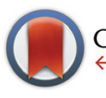

CrossMark « click for updates

Cite this: Food Funct., 2016, 7, 4041

\author{
M. A. Jimoh, ${ }^{a}$ W. MacNaughtan, ${ }^{a}$ H. E. L. Williams, ${ }^{b}$ D. Greetham, ${ }^{a}$ R. L. Linforth ${ }^{a}$ and \\ I. D. Fisk ${ }^{a}$
}

\section{Sodium ion interaction with psyllium husk (Plantago sp.) $\dagger$}

Received 27th May 2016 Accepted 18th August 2016

DOI: $10.1039 /$ c6fo000785f www.rsc.org/foodfunction

\begin{abstract}
The nature of and factors effecting sodium interactions with psyllium were investigated in vitro. In a batch extraction system, psyllium mucilage gel retained at least $50 \%$ of sodium across a range of concentrations (5-300 mg sodium per g psyllium) and pH (2-10) environments. FTIR and Na NMR analyses of psyllium gels indicated that binding was complex with non-specific multi-site interactions. The potential use of psyllium husk as a binding agent for the reduction of bioavailable sodium was therefore evaluated. The binding of sodium at physiologically relevant conditions ( $\mathrm{pH} 1.2$ (stomach) and 6.8 (intestine)) was studied in a gastrointestinal tract (GIT) $\mathrm{pH}$ simulated model. Results show consistently high sodium retention ( $50 \%$ ) across the GIT model and less than $20 \%$ loss of bound sodium under the simulated intestinal $\mathrm{pH}$ conditions after repeated washings.
\end{abstract}

\section{Introduction}

Since the introduction of the UK salt reduction program in $2003,{ }^{1}$ the consumption of salt in the population has been reduced by $15 \%$ according to data from the National Diet and Nutrition Survey. ${ }^{2}$ However with salt consumption at $\sim 8 \mathrm{~g}$ per day, salt consumption is yet to reach the recommended target of $5 \mathrm{~g}$ per day for adults. A high salt (sodium) diet increases the risk of high blood pressure which in turn increases the chance of having a heart attack or stroke. Reducing population salt intake by $1 \mathrm{~g}$ per day has been proposed to mitigate 4147 premature deaths and will save the National Health Service (NHS) £288 million annually. ${ }^{3}$ The World Health Organization (WHO) has pledged its support by developing tools for the identification of population salt consumption and major sources of sodium in the diet; support for the reformulation of a set number of products on the market; support in developing health communication material to increase consumer awareness on salt/sodium and to inform consumers on how to read and interpret food labels. ${ }^{4}$

Sodium has multiple roles within food and as such salt reduction strategies are required to be multifaceted. One

\footnotetext{
${ }^{a}$ Division of Food Sciences, School of Biosciences, University of Nottingham, Sutton Bonington Campus, Loughborough, Leicestershire LE12 5RD, UK. E-mail: Ian.fisk@nottingham.ac.uk; Fax: +44 (0)115 95 16142; Tel: +44 (o)1159516037

${ }^{b}$ Centre for Biomolecular Sciences, School of Chemistry, University of Nottingham, $U K$

$\dagger$ Electronic supplementary information (ESI) available. See DOI: 10.1039/ c6fo00785f
}

common approach is the replacement of sodium with similar salty tasting ions such as potassium and ammonium; unfortunately these salts carry additional flavours and aftertastes and therefore cannot be used as a direct complete replacement for sodium.

Sodium plays an anti-microbial and textural function in processed foods and the impact of sodium reduction on these functions has yet to be fully resolved. The use of flavour enhancers that amplify the perceived salty taste of sodium chloride, or replicate the flavour enhancing properties of sodium ions, include yeast extracts and specific nucleotide and glutamate preparations. Whilst these are partially successful, there is often an impact on production costs and require modifications to nutritional labels. One example being the use of seaweed as a potential salt replacer. ${ }^{5}$

A related approach to reduce sodium content in food is by gradual reduction of the salt content over an extended time period, typically months or years. ${ }^{6}$ This reduction by stealth requires industry-wide cooperation and long time periods to achieve significant reductions.

Most research approaches in our laboratories ${ }^{7-9}$ and indeed elsewhere ${ }^{10-12}$ have focused on optimising the delivery or bioaccessibility of sodium (e.g. through studies on mitigating carriers to dissolution, diffusion and effecting mixing).

The work presented here aims to tackle the issue of salt reduction in the diet through modifying the (bio) availability of sodium (molecular binding). Our current hypothesis is that the binding of sodium to natural products such as polysaccharides (especially indigestible dietary fibres) would serve to reduce dietary sodium bioavailability and increase sodium excretion through the faecal route. 
Natural polysaccharides like other biopolymers, have gained interest in the food and pharmaceutical industries due to their versatility and biocompatibility/biodegradability. They have found use as stabilizers, thickeners, binders, dispersants, lubricants, adhesives and drug-delivery agents, amongst other applications. There is ongoing interest in the use of plant based polysaccharides as a functional food ingredient in the prevention and treatment of obesity, diseases such as cardiovascular disease and cancer e.g. (ref. 13) and references therein.

Psyllium (Plantago sp.) seeds and husk have gel forming and dietary bulking properties due to the seeds being coated in an external polysaccharide mucilage layer (husk). Psyllium husk has been used as a laxative for the treatment of irritable bowel disorder (IBD) symptoms and as a health supplement. Psyllium husk and its derivatives have also found application for targeted drug release e.g. as a vehicle for the targeted delivery to the colon. ${ }^{14}$ Environmental applications have included its use as natural flocculation agent in textile wastewater remediation $^{15}$ and as a heavy metal ion scavenger. The mucilage from psyllium husk (Plantago ovata) is mainly composed of arabinose and xylose sugars in a highly branched polymer configuration. ${ }^{16-21}$

The aim of this study was to evaluate psyllium husk as a binding agent for the reduction of bioavailable sodium. The binding of sodium to psyllium was evaluated across a gastrointestinal tract (GIT) model to identify if psyllium husk has the potential to reduce total bioavailability of sodium.

\section{Materials and methods}

Psyllium samples (Elf Foods, Loughborough, UK), were stored in plastic air-tight sample containers, and used within their indicated shelf-life (psyllium seeds and husk Sidhpur SAT-ISABGOL Factory brand both packaged by East Foods Ltd).

Sodium chloride, potassium hydroxide, ammonium chloride, ammonium hydroxide, hydrochloric and nitric acid were at least Analar® grade ( $>98 \%$ purity) and obtained from Fisher Scientific, Loughborough UK. TraceMetal Grade nitric acid (evaluated for 65 metals $<1 \mathrm{ppb}$ ) was used for wet digestion prior to total trace element analysis. Ionic strength adjuster (ISA) was $21 \%$ ammonium chloride in $14 \%$ ammonium hydroxide solution.

\section{Elemental analysis and material characterisation by FTIR}

Total sodium, potassium, calcium and magnesium content were determined by inductively coupled plasma atomic emission spectrometry (ICP-AES) using a Perkin Elmer Optima 2000 DV after microwave digestion (Anton Paar microwave oven) of $200 \mathrm{mg}$ psyllium seed and husk samples with concentrated nitric acid $(6 \mathrm{~mL})$. The digests were diluted with ultrapure water to $40 \mathrm{~mL}$ and analysed for sodium $(589.592 \mathrm{~nm}$ line), potassium $(766.49,404.721 \mathrm{~nm}$ lines), calcium (315.887, $317.933 \mathrm{~nm}$ lines) and magnesium (279.077, $285.213 \mathrm{~nm}$ lines). Analysis was in triplicate with reagent blanks.
Quantification was against standards run in the concentration range of $0-20 \mathrm{ppm}$.

Operationally defined water extractible sodium fraction and acid extractible fraction were determined by carrying out parallel extractions of $500 \mathrm{mg}$ of psyllium seed and husk samples with $5 \mathrm{~mL}$ of ultrapure water $(18.2 \mathrm{M} \Omega$ ) and $0.02 \mathrm{M}$ hydrochloric acid respectively and analysis of extracts obtained for sodium.

CHN analysis of psyllium seed and husk samples was carried out on $2 \mathrm{mg}$ samples by an external laboratory (Laboratory Services, Chemistry Department, University of Nottingham) using a Perkin Elmer CHN analyser.

Fourier Transform Infra-red Spectroscopy (FTIR) measurements were carried out using a Tensor 27 spectrometer (Bruker, Coventry UK) equipped with a deuterated triglycine sulfate (DTGS) detector in attenuated total internal reflectance (ATR) mode. Single spectra of ground seed (355 $\mu \mathrm{m})$ and husk samples, as well as monosaccharide standards (arabinose, xylose and galactose) and xylan were recorded between 600 and $4000 \mathrm{~cm}^{-1}$, at $4 \mathrm{~cm}^{-1}$ resolution and resulted from the coaddition of 128 scans. The raw spectra were baseline corrected and vector normalised using the OPUS software.

\section{Evaluation of sodium interaction with psyllium}

For estimation of bound fraction, a general extraction procedure was developed in which $500 \pm 1 \mathrm{mg}$ of sample was shaken with $5 \mathrm{~mL}$ of test solution containing a known amount of sodium. $5 \mathrm{~mL}$ of ultrapure water or aqueous surfactant solution (Sherwood photometer diluent solution) was added and the mixture shaken for $30 \mathrm{~min}$ and solids separated by centrifugation at $412 g$ rpm for 20 min (multi-function centrifuge, Thermo Electron Corp.). The supernatant was removed for instrumental analysis to estimate free sodium. Sodium analysis was by flame photometry (Flame photometer Model 410 Sherwood Scientific Ltd, Cambridge, UK) or potentiometry (Ion meter (Jenway pH meter 3023) with sodium ion selective electrode (Cole-Parmer, London UK)). For flame photometric analysis, $2 \mathrm{~mL}$ of sample (supernatant) was diluted as required using the diluent solution to be in the linear range 1-10 ppm of the instrument. A direct comparison between sample reading and those of calibration standards gave the concentration of sodium. For potentiometric analysis, $200 \mu \mathrm{L}$ of ionic strength adjuster (ISA) was added to $2 \mathrm{~mL}$ of supernatant and the mixture analysed. Standards of same volume were also analysed after addition of ISA. Bound sodium was calculated as difference between total sodium added and the free sodium measured. \% Retention or \% bound sodium was calculated from eqn (1) below

$$
\% \text { Bound }=100 \times(\text { total-free }) \text { sodium } / \text { total sodium }
$$

\section{Influence of sample preparation}

To investigate the influence of particle size, type of grinding and properties of seed on the interaction of Plantago sp. with sodium, seed samples were ground using either a laboratory 
disc-mill (Perten AB disc mill LM 3310, Hägersten Sweden) for 'milled' sample or a coffee grinder (DeLonghi KG series, Hampshire, UK) for 'ground' sample. The ground material was sieved through a series of laboratory sieves (Endecotts Ltd, London, England) of mesh sizes 106-355 $\mu \mathrm{m}$ and size fractions collected. Psyllium husk samples were used as obtained. Hydrogels were developed by adding $100 \mathrm{~mL}$ of ultrapure water to 1-2 $\mathrm{g}$ of psyllium husk. The mixture was dried overnight at $40^{\circ} \mathrm{C}$, ground and carried through sodium binding experiments for comparison.

\section{Evaluation of the role of $\mathrm{pH}$ on sodium interaction}

To study the effect of $\mathrm{pH}$ on the retention of sodium by psyllium husk, the general extraction procedure described above was followed except that the $\mathrm{pH}$ of test $\mathrm{Na}$ standard solutions $(0.1 \% \mathrm{w} / \mathrm{w})$ was adjusted to $2,4,6,8$ and 10 using dilute hydrochloric acid and potassium hydroxide solutions. Final $\mathrm{pH}$ of the mixture was checked with a pH meter (InoLab Level 1 pH meter, VWR Germany).

\section{Analysis of binding capacity}

To estimate binding capacity, $500 \mathrm{mg}$ of psyllium husk was mixed with $25 \mathrm{~mL}$ of solutions with various starting sodium concentrations (5-300 $\mathrm{mg} \mathrm{g}^{-1}$ ) and the general extraction procedure applied as before.

\section{Analysis of sodium retention at physiologically relevant $\mathbf{p H}$}

To test the theoretical suitability of psyllium husk (Plantago sp.) as a scavenger of sodium in food, the retention behaviour of psyllium at the physiologically relevant $\mathrm{pH}$ of 1.2 for the stomach and 6.8 for the small intestine was evaluated. In this experiment, synthetic gastric juice formulations and buffers were avoided as they contained high levels of sodium. Thus $500 \mathrm{mg}$ of husk sample was mixed with $5 \mathrm{~mL}$ of $0.1 \% \mathrm{w} / \mathrm{w}$ test sodium solution and $20 \mathrm{~mL}$ of ultrapure water. The mixture was centrifuged $(412 g)$ and an aliquot of the supernatant taken for analysis. The $\mathrm{pH}$ of the remaining mixture was adjusted to pH 1.2 using hydrochloric acid, thus simulating stomach $\mathrm{pH}$ conditions. The mixture was again centrifuged $(412 g)$ and an aliquot of the supernatant taken for sodium analysis. Thereafter, a further $5 \mathrm{~mL}$ of $0.1 \% \mathrm{w} / \mathrm{w}$ sodium solution was added, (to adjust the final volume and simulate the reflux of sodium in the intestine), then the $\mathrm{pH}$ was adjusted to 6.8 with dilute potassium hydroxide solution, simulating $\mathrm{pH}$ conditions in the intestine. This mixture was separated by centrifugation as before and the concentration of sodium in the supernatant determined as before.

To test the release of 'psyllium bound' sodium in the intestine, $5 \mathrm{~mL}$ of a $1 \% \mathrm{w} / \mathrm{w}$ sodium solution was added to $500 \mathrm{mg}$ psyllium husk (100 mg sodium per $\mathrm{g}$ psyllium), shaken and the resulting 'sodium loaded' psyllium husk gel mixed with $20 \mathrm{~mL}$ of ultrapure water. After centrifugation, the supernatant was removed and sodium content determined. Further washings with $10 \mathrm{~mL}$ portions of ultrapure water followed. Sodium content in each washing step was also determined and compared.

\section{Analysis of psyllium gels by FTIR}

To investigate the nature of interaction and binding sites of sodium in psyllium, FTIR-ATR spectra of psyllium husk gels prepared under different treatment conditions were obtained using a Bruker spectrometer as previously described. Gels were prepared by adding $5 \mathrm{~mL}$ of test solution to $500 \mathrm{mg}$ of husk and shaking (30 min). Acid and alkali treatment gels were prepared by adding $0.1 \mathrm{M} \mathrm{HCL}$ and $0.1 \mathrm{M} \mathrm{KOH}$ to husk samples respectively. For the sodium treatment gel, 1\% (w/v) sodium solution was added. Sodium-calcium mixed gels contained $2.5 \mathrm{~mL}$ of $1 \%(\mathrm{w} / \mathrm{v})$ sodium and $1 \%(\mathrm{w} / \mathrm{v})$ calcium solutions. Calcium treatment gel contained $1 \%$ calcium solution. A water gel control was prepared by mixing $500 \mathrm{mg}$ husk and $5 \mathrm{~mL}$ ultrapure water, a further sample control of sodium chloride solution was taken.

\section{Analysis of sodium binding by ${ }^{23} \mathrm{Na}-\mathrm{NMR}$}

Na-NMR spectra were recorded using a R4-1004 compact Na-NMR spectrometer (AMR Ltd, Oxfordshire). Scans (4k) were recorded with a $90^{\circ}$ pulse width of $20 \mu$ s and a relaxation delay of $100 \mathrm{~ms}$. For the experiments, psyllium gels prepared in $0.1-5 \%(\mathrm{w} / \mathrm{w})$ sodium chloride solutions were weighed into NMR tubes and left to equilibrate at $20{ }^{\circ} \mathrm{C}$ prior to measurement. Standard sodium chloride solutions and blanks were also analysed.

$\mathrm{Na}$ single quantum (SQ) and double quantum filtered (DQF) NMR experiments ${ }^{22}$ were recorded at $158 \mathrm{MHz}$ on a $600 \mathrm{MHz}$ Bruker Avance III spectrometer equipped with a $5 \mathrm{~mm}$ broadband probe. Samples contained D2O (1:10) to obtain a lock signal. DQ coherences were selected using a phase cycled pulse sequence. Optimum creation time for maximum amplitude of the DQ signal was found by locating the maximum intensity for spectra acquired at different creation times.

\section{Data analysis}

Statistical data analysis was carried out using the GraphPad Prism 6 statistical package (GraphPad Software, Inc.), Origin (OrignlabInc) and Excel (Microsoft Corp). Experiments were carried out in triplicate and values reported are means \pm standard deviation.

\section{Results and discussion}

\section{Physico-chemical characterisation and binding capacity}

Results of elemental analyses are given in Table 1 . While the sodium and potassium content of husk was higher than that of seed samples, the nitrogen content of husk was negligible compared to that of seed, indicating no amide groups were likely to be involved in sodium binding in the husk.

The binding of sodium to psyllium husk was evaluated in static batches; sodium was 'lost' from the psyllium gel after separation, indicating 69\% sodium retention (Table 2). In order to identify physical fractions with optimum sodium binding, a range of samples were prepared (seed, gel, husk) 
Table 1 Elemental composition of psyllium seed and husk

\begin{tabular}{lll}
\hline Element & Seed & Husk \\
\hline Carbon $^{a}$ & 44 & 40 \\
Hydrogen $^{a}$ & 7 & 6 \\
Nitrogen $^{a}$ & 2.7 & 0.1 \\
Sodium $^{b}$ & $35 \pm 2$ & $90 \pm 2$ \\
Potassium $^{b}$ & $2774 \pm 195$ & $6903 \pm 177$ \\
Magnesium $^{b}$ & $669 \pm 37$ & $59 \pm 2$ \\
Calcium $^{b}$ & $1454 \pm 58$ & $1413 \pm 10$ \\
${ }^{a}$ Mean \% value. &
\end{tabular}

Table 2 Effect of particle size and sample preparation on sodium binding in psyllium

\begin{tabular}{ll}
\hline Sample & \% Sodium retention \\
\hline Fraction size & \\
Whole seed & $29 \pm 2$ \\
$212-250 \mu \mathrm{m}$ & $51 \pm 3$ \\
$106-212 \mu \mathrm{m}$ & $77 \pm 0.3$ \\
$<106 \mu \mathrm{m}$ & $71 \pm 5$ \\
& \\
Sample preparation & \\
Coarse grind & $55 \pm 3$ \\
Psyllium gel & $69 \pm 1$ \\
Husk & $69 \pm 0.3$
\end{tabular}

$\%$ Sodium retention is $100 \times$ (bound sodium/added sodium), $\mathrm{mg} \mathrm{g}^{-1}$ husk \pm standard deviation; added sodium was $10 \mathrm{mg} \mathrm{g}^{-1}$ psyllium.

with a range of grind sizes (whole seed to finely ground material, $<212 \mu \mathrm{m}$ ), their ability to bind to sodium in a batch reactor was evaluated (Table 2).

Particle sizes fractions of the seed of less than $212 \mu \mathrm{m}$ had the best retention under the experimental conditions tested. This is to be expected as the smaller particle size fractions would have a greater number of broken cells with a larger proportion of exposed intra-cellular contents ultimately creating a larger surface area to facilitate sodium interaction. Van Craeyveld et al. ${ }^{23}$ reported the increased solubility and accessibility of arabinoxylan in ball-mill treated husk samples compared with untreated samples. As the retention of sodium in psyllium husk was about the same as the finely ground seed $(<216 \mu \mathrm{m}$ fraction), psyllium husk as received, was used for the rest of the studies (i.e. without further preparation). In the following discussions, psyllium, psyllium gel and husk are used interchangeably to mean samples prepared from psyllium husk.

The influence of $\mathrm{pH}$ on the binding of sodium by the psyllium husk

The influence of $\mathrm{pH}$ on sodium binding was studied over a range of $\mathrm{pH}^{\prime}$. $^{2-10}$ The binding of sodium by psyllium husk varied slightly as a function of $\mathrm{pH}$, as a slightly higher retention was observed under acidic conditions $(\mathrm{pH} 2)$ than at near neutral $(\mathrm{pH} 6$ or $\mathrm{pH}$ 8) or alkaline $\mathrm{pH}$ conditions ( $\mathrm{pH} 10)$ (Fig. 1). This slight increase in retention at low $\mathrm{pH}$ could be due to changes in charge state, modification of hydration

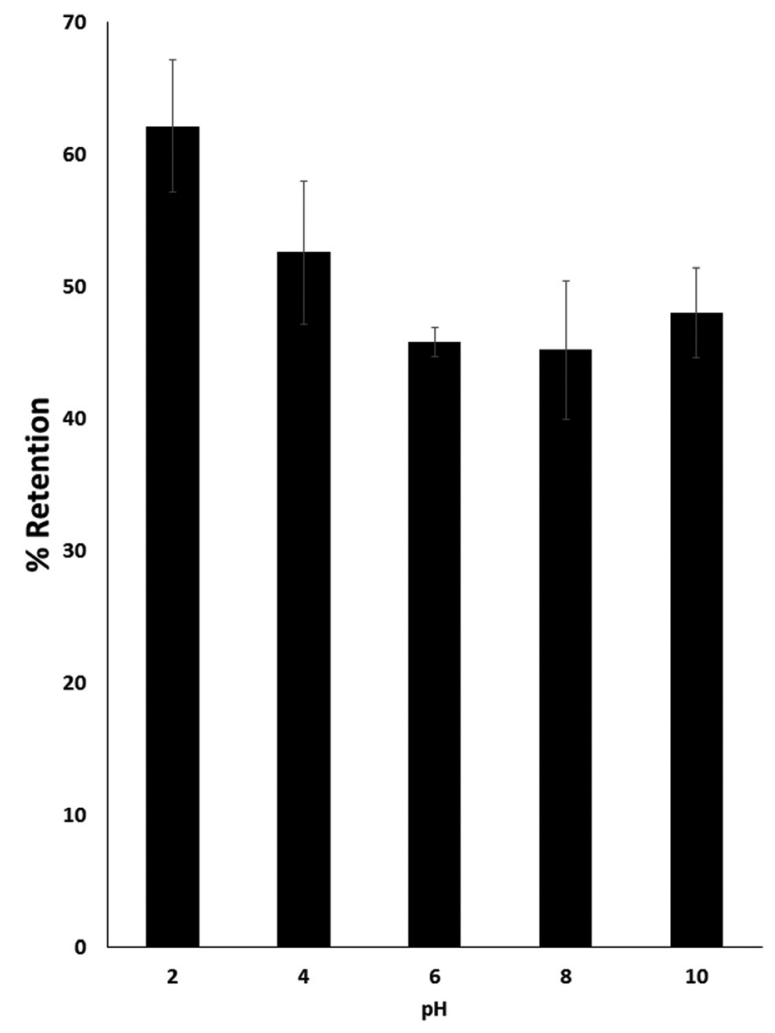

Fig. 1 Influence of $\mathrm{pH}$ on sodium binding by psyllium husk. Gels were developed by mixing $5 \mathrm{~g}$ husk with $5 \mathrm{~mL}$ of $0.1 \%$ sodium solution, these were then adjusted to the required $\mathrm{pH}$ with $\mathrm{HCl}$ or $\mathrm{KOH}$ solution.

shells or morphological changes of the biopolymer at acidic conditions. Indeed, the streaming potential of psyllium husk gel prepared in acid ( $\mathrm{pH} 2$ ) was at least 10 times less negative than those prepared in alkali $(\mathrm{pH} 11)$ or without $\mathrm{pH}$ adjustment ( $\mathrm{pH}$ 6.7). Furthermore, charge density of psyllium gels increased as alkalinty increased from acidic gels $(\mathrm{pH} 2)$ to neutral ( $\mathrm{pH}$ 6.7) to alkali ( $\mathrm{pH}$ 11), indicating $\mathrm{pH}$ drives changes to the hydration shell and supports the hypothesis that sodium interaction may occur partially by a charge based mechanism. See ESI $\uparrow$ for streaming potential method details.

\section{Influence of pH as studied by FTIR spectroscopy}

FTIR spectra of psyllium gels prepared in acid $(0.1 \mathrm{M} \mathrm{HCl})$ solutions showed distinct differences from those prepared in sodium (1\%) solutions in the fingerprint region $1200-700 \mathrm{~cm}^{-1}$, O-H bands about 1630 and $3600-3300 \mathrm{~cm}^{-1}$. Subtraction of the acid spectra from those of alkali gels prepared in $0.1 \mathrm{M} \mathrm{KOH}$ solution showed major peak intensity differences at 693, 1049 and $1734 \mathrm{~cm}^{-1}$. The 2nd derivatives further revealed the appearance of a peak at 945 and 892 for the acid gel only in the $\beta$-pyranose 980-880 region indicating different interactions of sodium or reduced accessibility to the ring. Slight differences in the form of shifts to higher wavenumbers in 1740-1600 regions in the acid gel compared to the sodium and other gels were also observed. This indicates a strong interaction of acid with the psyllium husk; increased 


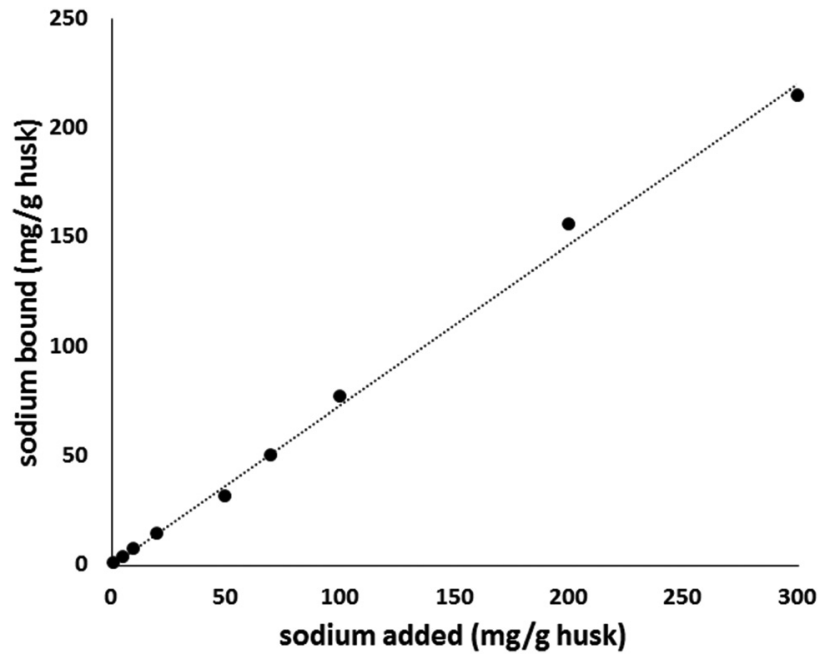

Fig. 2 Effect of sodium concentration on sodium binding of psyllium $500 \mathrm{mg}$ of psyllium husk was mixed with solutions containing increasing concentrations of sodium.

accessibility could explain the slight increase in sodium retention found at lower $\mathrm{pH}$ conditions within the batch isotherm experiments. The binding mechanism and possible binding sites were evaluated using FTIR and Na-NMR and are discussed below. Fig. 2 shows the binding behaviour of psyllium husk with different starting concentrations of sodium. Concentrations of sodium below $50 \mathrm{mg} \mathrm{g}^{-1}$ husk followed the Langmuir model (see ESI $\dagger$ ), indicating a monolayer adsorption mechanism. At sodium concentrations higher than $100 \mathrm{mg} \mathrm{g}^{-1}$ husk, complex heterogeneous adsorption is assumed to take place through secondary layers. This would explain the high percentage of sodium (at least 50\%) retained across the range of concentrations studied.

\section{Sodium retention at physiologically relevant $\mathrm{pH}$}

Sodium retention at physiologically relevant pH 1.2 and 6.8 was tested in order to assess the retention performance of psyllium in the human gastrointestinal tract. Due to the high sodium content of synthetic gastric juice and buffers available, test solutions containing known amounts of sodium adjusted to relevant $\mathrm{pH}$ were used instead. Samples were held at $\mathrm{pH}$ values relating to the mouth, stomach and intestine to simulate digestion. It is assumed that most of the sample passes from the mouth to the stomach, at this stage, $47 \%$ of the sodium was shown to be retained within the gel (Table 3). If the model is continued further to the intestine, the sodium retention was $47 \%$ indicating a significant proportion of sodium is retained and not biologically available. To simulate the effect of sodium reflux into the intestine on the binding capability of psyllium, a further addition of $5 \mathrm{~mL}$ of $0.1 \% \mathrm{w} / \mathrm{w}$ sodium test solution was made into the GIT model system (Table 3). The extended GIT model showed that the introduction of a fresh flux of sodium in the intestine did not adversely affect the binding ability as seen in the last step of the simu-
Table 3 Results of sodium binding at simulated gastrointestinal tract $\mathrm{pH}$. A second aliquot of sodium ions were added to the model to simulate sodium reflux into the intestine

\begin{tabular}{lrl}
\hline Model & $\mathrm{pH}$ & \% Sodium retention \\
\hline In-mouth & $\sim 6.5$ & $47 \pm 1$ \\
Stomach & 1.2 & $45 \pm 1$ \\
Intestine & 6.8 & $47 \pm 1$ \\
Sodium addition & & \\
Intestine & 6.8 & $58 \pm 6$
\end{tabular}

$\%$ Retention is calculated as \% bound sodium/added sodium $\pm \mathrm{sd}$.

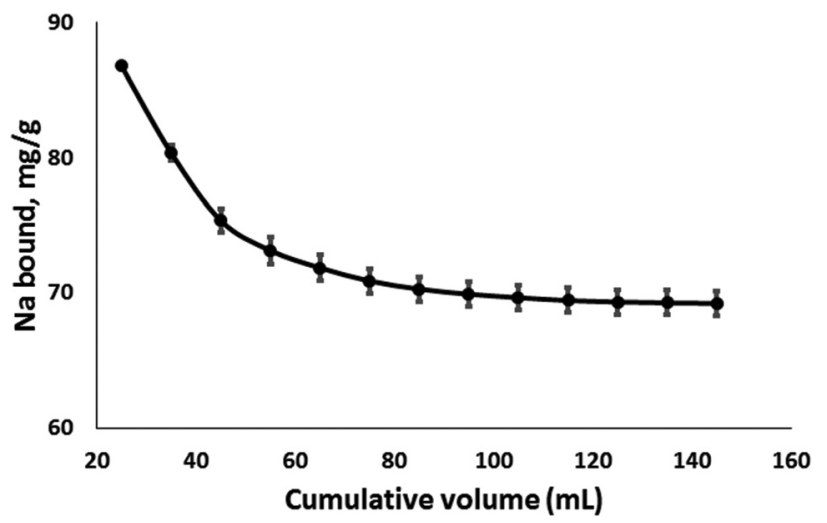

Fig. 3 Stability of psyllium bound sodium under repeated washings. Starting concentration was $50 \mathrm{mg} \mathrm{Na}$ per $0.5 \mathrm{~g}$ husk (washing with $10 \mathrm{~mL}$ aliquots of ultrapure water).

lated GIT model. These results indicate that psyllium could be a potential control mechanism for sodium bioaccessibility.

Due to the production of digestive fluids and intake of liquid foods, release of bound sodium under intestinal $\mathrm{pH}$ conditions was studied with repeat washings. The results showed that over $80 \%$ of the initially bound sodium was retained after 12 repeated washings using a total volume of $120 \mathrm{~mL}$ (Fig. 3).

\section{Further FTIR study on sodium interaction in psyllium gels}

FTIR spectra were generated of psyllium gels prepared under different treatment conditions to propose possible sites of interaction. There were no gross differences between the native gel and the gel with sodium $(P>0.05$, Fig. 4$)$. As expected a peak at $1044 \mathrm{~cm}^{-1}(\mathrm{C}=\mathrm{O})$ was not present in the deionised water and sodium chloride blank solutions. However, peak shifts in the $3600-3100 \mathrm{~cm}^{-1}, 1650-1630 \mathrm{~cm}^{-1}$ and $690-600 \mathrm{~cm}^{-1}$ regions were further investigated using subtraction, random forest classification analysis and analysis of 2nd derivatives. Subtraction of different treatments from the sodium gel spectra showed interaction with sodium at $3500-3300,1080-1040$ and the arabinoxylan backbone at 690-600 $\mathrm{cm}^{-1}$ indicating multi-site, non-specific interactions probably with hydroxyl and carboxylic acid groups.

Random forest classification confirmed that wavenumbers ranked in order of significance were the free H-bonding region 


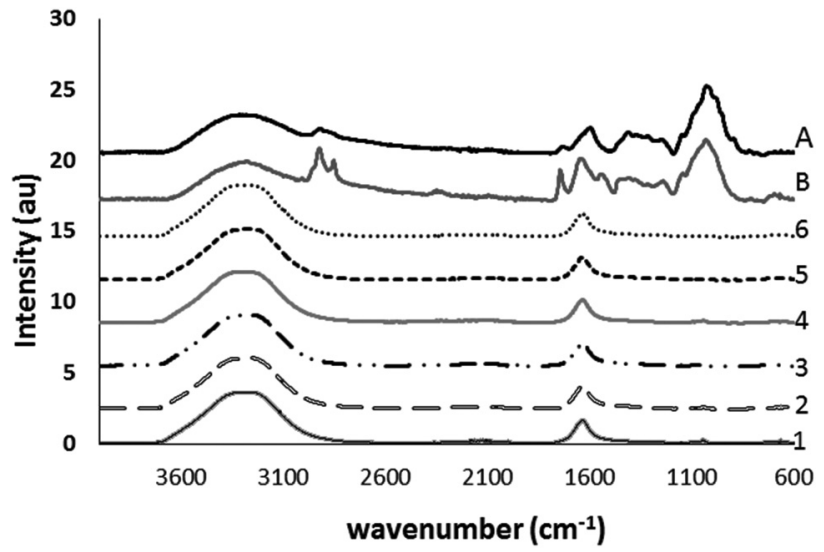

Fig. 4 FTIR spectra of psyllium gels prepared under different treatments. 1. Psyllium gel in acid, 2. Psyllium gel in alkali, 3. Psyllium gel in water, 4. Psyllium gel containing sodium, 5. Deionised water and 6. $2 \%$ salt solution. Spectra of native husk (A) and seed (B) are added for comparison.

$3997-3600$, OH region of $1750-1600$ and the $700-600 \mathrm{~cm}^{-1}$ polymer region.

Spectra presented as 2nd derivative also illustrated changes in the $680-660 \mathrm{~cm}^{-1}$ region which relates to the arabinoxylan backbone, this was similar for the water gel and the calcium containing gel. A shift to lower wavelengths indicated a weakening of bonds or greater interactions and was seen for sodium, acid and alkali treatments in the order sodium $\gg$ acid $>$ alkali.

It must be borne in mind that the assignments of bands is not absolute and therefore Na-NMR experiments were carried out to further understand the mechanism of sodium binding within the psyllium gel.

\section{${ }^{23}$ Na-NMR studies on sodium binding mechanism}

Two approaches were followed in the ${ }^{23} \mathrm{Na}$-NMR study. In the 1st approach, free induction decay (FID) data obtained from runs of sodium standards and corresponding psyllium gels containing known but varying amounts of sodium were compared. The difference in measurable sodium can be used to indicate free sodium and bound sodium fractions. The results indicated that approx. $10 \%$ sodium is unmeasurable by NMR and is presumed to be strongly interacting (Fig. 5a). Since a higher binding affinity was observed in other experiments, the remaining bound fraction was assumed to be less tightly interacting with the biopolymer matrix, presumably through a hydration shell or steric interaction mechanism. However, we suspect that instrumental limitations (significant dead time of $50 \mu \mathrm{s}$ for the probe), caused uncertainty in the measurement of the initial amplitude of the signal. Since the decay time for the signal decreases with increasing binding (see ESI Table $1 \dagger$ ), the decay time values of the psyllium gel experiments may be biased. This begs the question of how bound is this bound fraction or 'lost' to the NMR fraction. The psyllium gel and the salt solution are essentially "liquid like" samples,
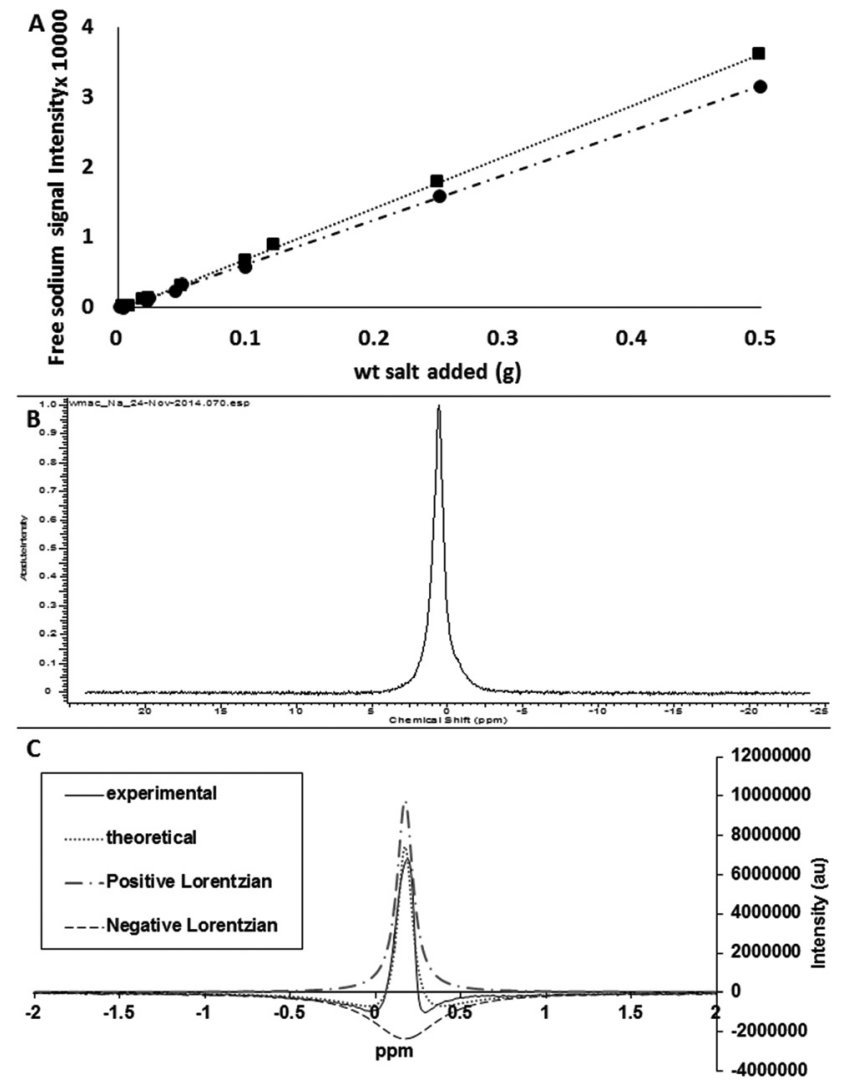

Fig. 5 (A) Free sodium chloride detected by compact Na-NMR in standard sodium chloride solutions (square) and the corresponding concentrations of sodium chloride in psyllium gels (circle). Gels were prepared from $500 \mathrm{mg}$ husk in $5 \mathrm{~mL}$ of the appropriate concentration of sodium chloride solution (top); (B) single quantum spectrum from psyllium gel prepared in $2 \%$ sodium chloride solution (middle); (C) example double quantum filtered signal generated from a carrageenan sample prepared in $2 \%$ sodium chloride solution using the same pulse sequence as for psyllium gel samples (no signal), and fit with double Lorentzian functions (bottom).

but can exhibit a range of mobility and can generate different Na-NMR spectra due to the different physical phenomena involved. In the simple FID measurement as in the benchtop compact NMR described in the 1st approach, only the free tumbling ions can be measured.

In the 2nd approach, a high resolution $600 \mathrm{MHz}$ NMR instrument afforded advanced quantum filtration experiments to measure those sodium nuclei which are experiencing a restriction in their motion and so distinguish the sodium ions in different environments(-isotropic/anisotropic). In a normal single quantum experiment, the satellite lines of the spectral triplet are broadened and this produces a double exponential decay in the single quantum FID or equivalently a broadened base in the spectral line (Fig. 5b). When passed through the double quantum filter the result is two antiphase Lorentzian lines as can be seen in the $2 \%$ sodium content carrageenan example in Fig. 5c. For psyllium gels with a $0 \%, 0.5 \%$ and $2 \%$ sodium content, no DQF signal was observed, indicating no 
strongly bound fraction of sodium within the gels. This points ultimately towards a non-specific occlusion mechanism.

\section{Conclusions}

Sodium was shown to bind to psyllium through a multi-site, non-specific mechanism. A tightly bound fraction is proposed to interact via mono layer adsorption, and a loosely bound fraction by a secondary occlusion process is postulated. The feasibility of psyllium as a scavenger of excess sodium in the diet has also been demonstrated. Psyllium is able to retain sodium across a range of $\mathrm{pH}$, including $\mathrm{pH}$ conditions of physiological importance to digestion, and across a wide sodium concentration range. Furthermore binding capacity is not dependent on sodium loading. Psyllium therefore shows promise as a means of reducing the bioavailable fraction of ingested sodium in the body. The biological significance of the findings should be further investigated in-body, in the presence of additional, biologically relevant, counter-ions.

\section{Acknowledgements}

ERDF, BBSRC (BB/N021126/1, BB/F017014/1) and the Food and Drink INET are acknowledged for funding this research. Dr J. Reps of the Advanced Data Analysis Centre (ADAC) University of Nottingham, provided FTIR data analysis support.

\section{References}

1 L. A. Wyness, J. L. Butriss and S. A. Stanner, Public Health Nutr., 2012, 15, 254-261.

2 F. J. He, H. C. Brinsden and G. A. MacGregor, J. Hum. Hypertens., 2014, 28, 345-352.

3 D. o. Health, New salt targets: F9 Salt Reduction 2017 pledge \& F10 Out of Home Salt reduction pledge. https:// responsibilitydeal.dh.gov.uk/responsibility-deal-food-networknew-salt-targets-f9-salt-reduction-2017-pledge-f10-out-ofhome-salt-reduction-pledge/, accessed 16th May 2014.
4 WHO, Global Strategy on Diet, Physical Activity and HealthPopulation sodium reduction strategies http://www.who. int/dietphysicalactivity/reducingsalt/en, accessed 16th May 2014.

5 EUTasteProject, The Application of Edible Seaweed for Taste Enhancement and Salt Replacement http://tasteproject.net, accessed 12th September 2014.

6 S. Girgis, B. Neal, J. Prescott, J. Prendergast, L. S. Dumbrel, C. Turner and M. Woodward, Eur. J. Clin. Nutr., 2003, 57, 616-620.

7 C. Morris, A.-L. Koliandris, B. Wolf, J. Hort and A. J. Taylor, Chemosens. Percept., 2009, 2, 1-8.

8 R. Rama, N. Chiu, M. Carvalho Da Silva, L. Hewson, J. Hort and I. D. Fisk, J. Texture Stud., 2013, 44, 338-345.

9 X. Tian and I. D. Fisk, Food Funct., 2012, 3, 376-380.

10 J. L. Busch, C. Tournier, J. E. Knoop, G. Kooyman and G. Smit, Chem. Senses, 2009, 34, 341-348.

11 M. W. J. Noort, J. H. F. Bult, M. Stieger and R. J. Hamer, J. Cereal Sci., 2010, 52, 378-386.

12 S. M. Goh, B. Leroux, C. A. Groeneschild and J. L. Busch, J. Food Sci., 2010, 75, S245-S249.

13 C. G. Biliaderis and M. S. Izydorczyk, Functional food carbohydrates, CRC Press, 1st edn, 2006.

14 B. Singh, Int. J. Pharm., 2007, 334, 1-14.

15 A. Mishra, R. Srinivasan and R. Dubey, Macromol. Mater. Eng., 2002, 287, 592-596.

16 J. Sandhu and G. Hudson, Carbohydr. Res., 1981, 247-259.

17 M. H. Fischer, N. Yu, G. R. Gray, J. Ralph, L. Anderson and J. A. Marlett, Carbohydr. Res., 2004, 339, 2009-2017.

18 R. Laidlaw and E. Percival, J. Chem. Soc., 1949, 1600-1607.

19 J. Kennedy, J. Sandhu and D. Southgate, Carbohydr. Res., 1979, 75, 265-274.

20 Q. Guo, S. W. Cui, Q. Wang and J. Christopher Young, Carbohydr. Polym., 2008, 73, 35-43.

21 A. Farahnaky, H. Askari, M. Majzoobi and G. Mesbahi, J. Food Eng., 2010, 100, 294-301.

22 R. Kemp-Harper, S. Brown, C. Hughes, P. Styles and S. Wimperis, Prog. Nucl. Magn. Reson. Spectrosc., 1997, 30, 157-181.

23 V. Van Craeyveld and J. A. Delcour, J. Agric. Food Chem., 2008, 56, 11306-11311. 